\title{
Lifestyle medicine and adverse childhood experiences
}

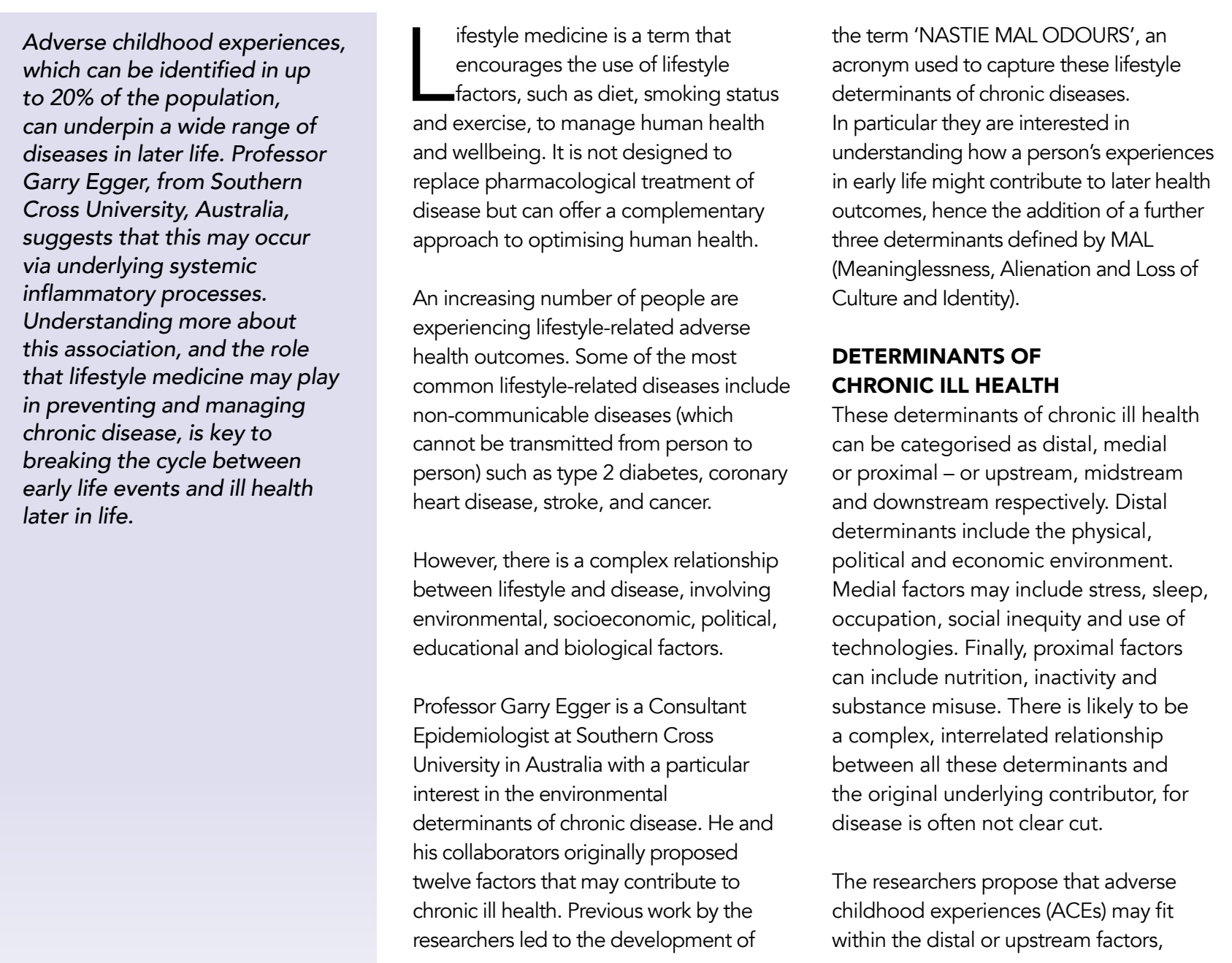

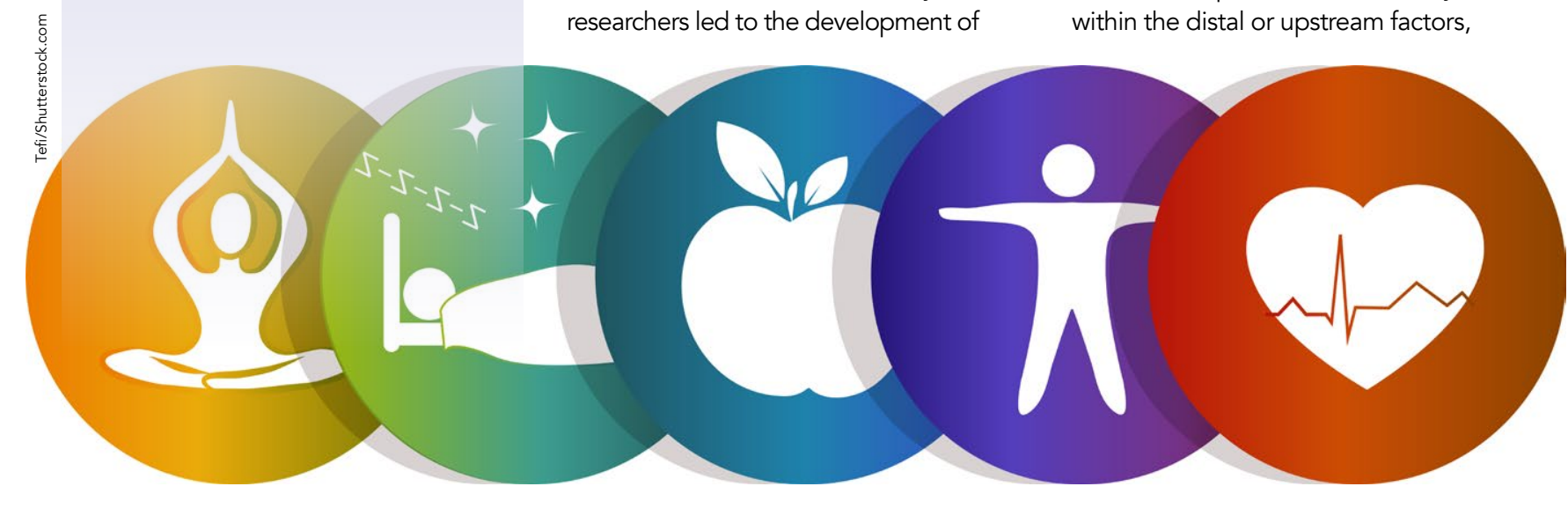

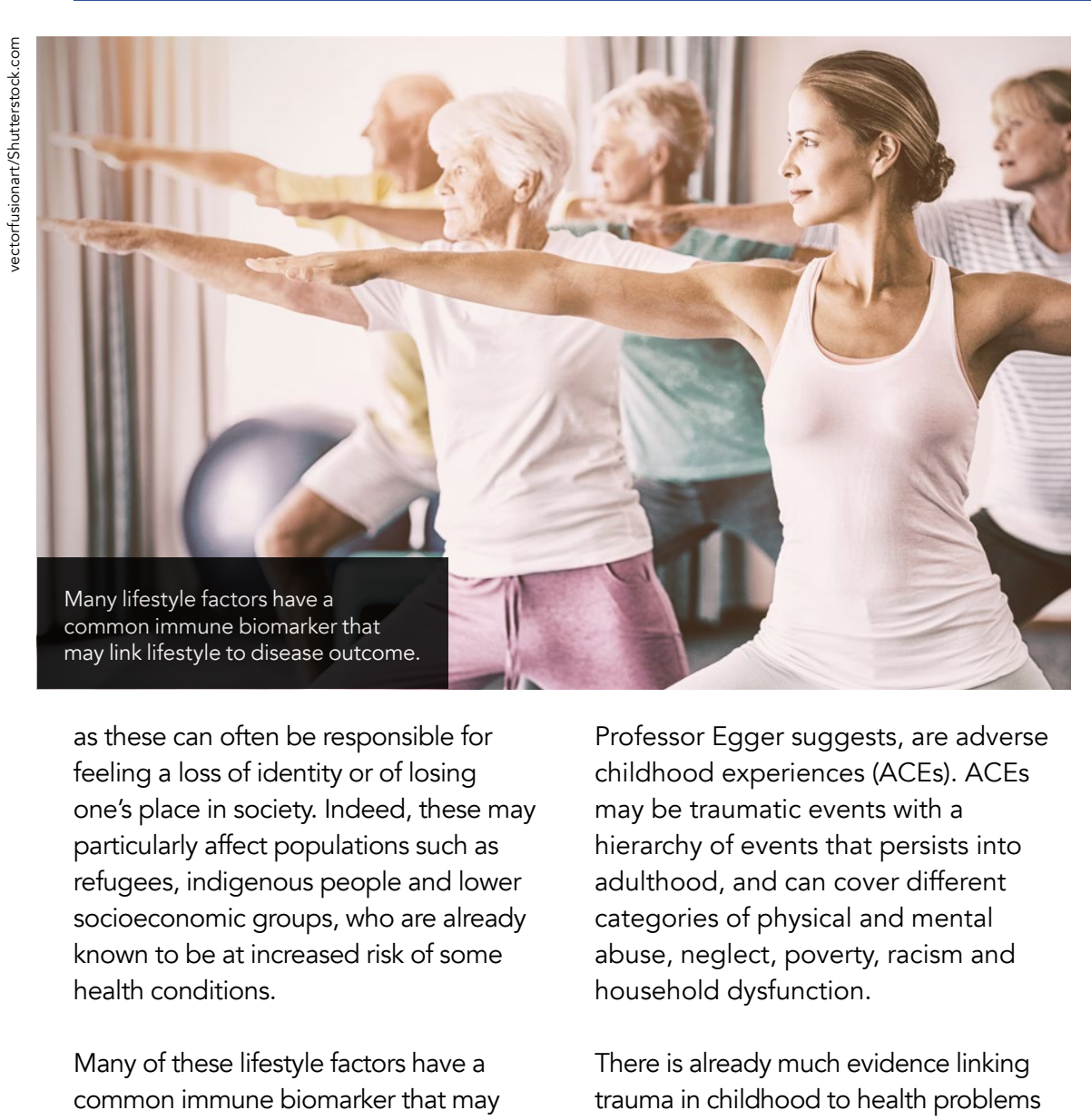

estyle factors, social disruption and . Understanding more about these preventative and man can shape how are implemented. To continue with the example of obesity, many traditional weight loss methods will be ineffective because of the coping strategies formulated in response to ACEs earlier in Iffe. A lifestyle-medicine-based approach requires a holistic view that takes into account health behaviour changes but also considers mental, emotional and psychological factors.

ACEs may impact health directly, that is independent of lifestyle, through conditions such as stress, anxiety and depression. However, ACEs may also factors. These psychological factors can be grouped together as meaninglessness, alienation and loss of culture and identity (MAL).

Professor Egger and his colleagues hypothesise that childhood experiences common immune biomarker that may trauma in childhood to health problems may act through MAL to influence chronic
link lifestyle to

disease outcome. $\quad$ Lifestyle medicine should be seen as
This is often called 'meta-flammation', a bridge between clinical medicine

which is a series

of inflammatory

events triggered by

the immune system, which can lead to a variety of metabolic disorders. It is also sometimes used to define a low-level, induced by metabolic factors. and public health. in later life. However, recent studies have illustrated that cumulative ACEs may have a bigger impact on health than single obesity and ACEs, likely facilitated by

PATHWAYS OF CHRONIC DISEASE As with obesity, many modern diseases are thought to be related to low-grade chronic inflammation. This systemic inflammation also appears to Professor Egger has previously suggested

Systemic, low-grade inflammation is often risk and allostasis. For example, chronic inflammation is known to occur in obesity and this inflammation can also have an impact on different body systems, such as the cardiovascular and renal system Allostasis maintains stability in the body by altering physiological parameters to counteract challenges. This is in contrast to homeostasis which keeps body systems, such as blood pH, within narrow operating ranges.

ADVERSE CHILDHOOD EXPERIENCES

One factor that may contribute to

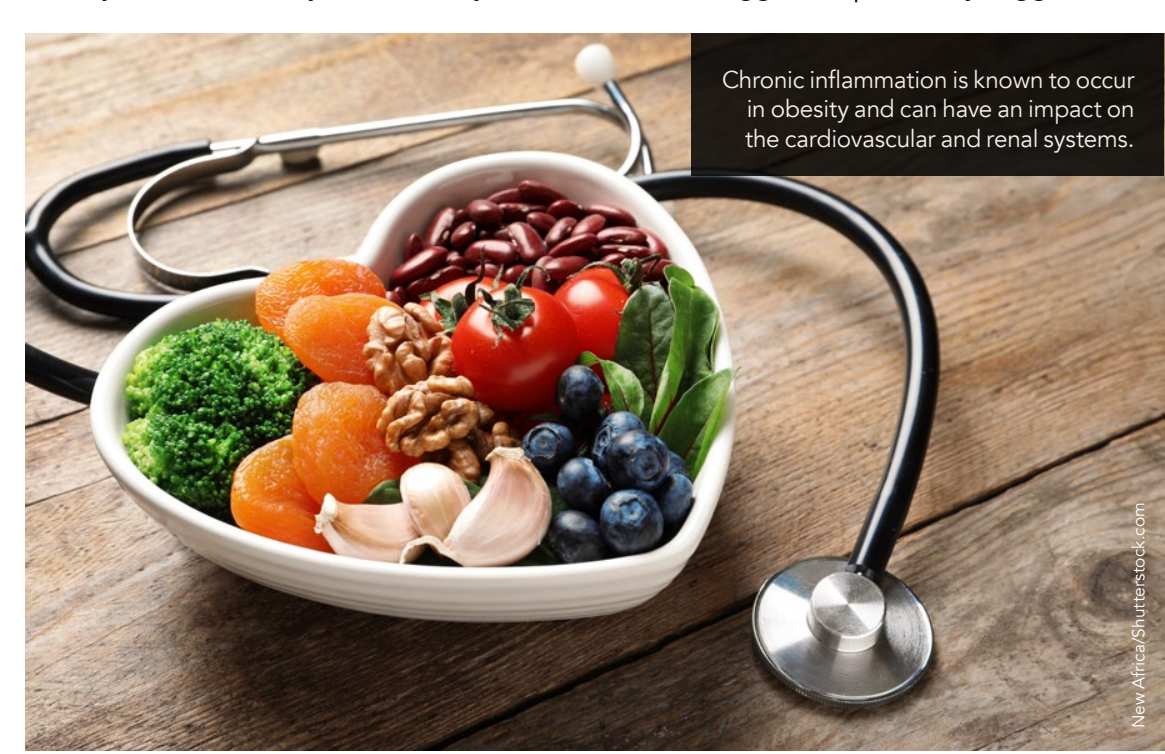




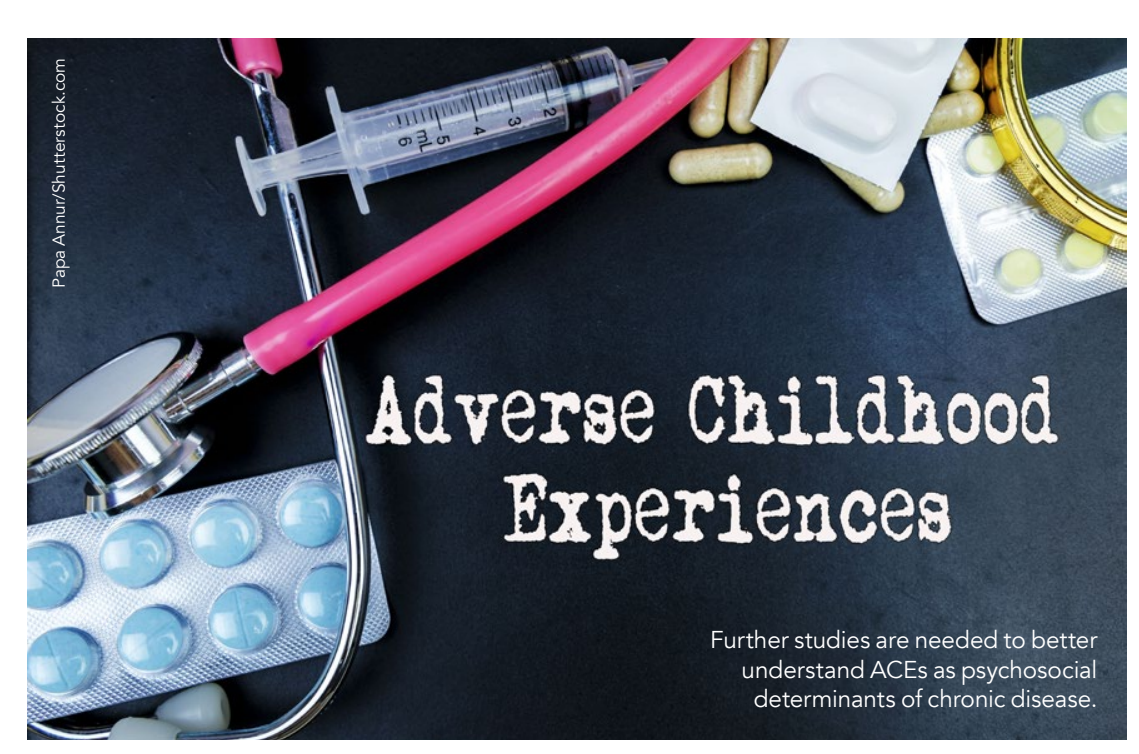

that exposure to specific environmental triggers can stimulate an immune metaflanmation As well tras the system, the nervous and endocrine systems may also be involved. The cumulative impact of these body-wide changes can cause a 'biological scar', which can in turn impact gene expression and regulation of physiological systems.

Biomarkers, molecules associated specifically with a certain disease or change in a biological system, have already been putatively identified in some long-term conditions stemming from ACEs. These biomarkers include molecules related to inflammatory processes, such as C-reactive protein (CRP) and interleukins. However, CRP is raised in many situations, including thection and non-specircintamation directly to chronic inflammation caused by lifestyle disease.

There is emerging evidence that supports the impact of ACEs on development of disease later in life and a number of different pathways have been proposed that may explain the epidemiology and neurobiology of the adverse effects of stress and trauma caused by ACEs.

\section{ACES AND LIFESTYLE MEDICINE} Health-related outcomes associated with ACEs include obesity, type 2 diabetes, ischemic heart disease, cancers, depression and decreased life span. Professor Egger highlights that disruption of the immune syste
observed as an increase in impact chronic disease outcomes. needed to better understand ACEs as psychosocial determinants of chronic disease, and to determine where lifestle He also points out that individuals will respond very differently to adverse experiences, as well as other confound factors such as gender genetics and possible interventions.

BENEFITS OF LIFESTYLE MEDICINE Professor Egger and his colleagues believe that lifestyle medicine should be seen as a bridge between clinical medicine and public health. They advocate a multifaceted approach to chronic, lifestyle related disease, including ACE-related disease. This should involve health promotion, social and cultural metaflammation, is an area that requires influence and legislation/regulation,
further research. He suggests that this
but also requires a level of individual metaflammation may develop early in responsibility. For example, MAL can be iffe and be part of longerterm changes treated with psychotherapy, cognitive development of other chronic diseases.

\section{These body-wide changes can cause}

a 'biological scar', which can in turn impact gene expression and regulation of physiological systems.

ACEs may also cause immune dysregulation though changes in health behaviours, either directly or indirectly, and these changes may have a further Alongside this, the influetaflammation. Alongside this, the influence of MAL (meaningless, alienation, or loss of culture

The causal pathway behind the relationship between ACEs and chronic disease is a highly complex web of interlinking factors. Professor Egger suggests that further studies are

to fully address it. In the context of ACEs, intervention is needed at a public health level to prevent chllahood abuse, but it also requires rehabilitation, such as culcural prescription, which is a form of social prescribing hat bings together arts, (n)

This is where lifestyle medicine may help, but in order to best target these interventions, further information about how ACEs, MAL and chronic inflammation interact to cause ill health may be required.

\section{LIFESTYLE DETERMINANTS OF CHRONIC DISEASE}

\begin{tabular}{|c|c|}
\hline \multicolumn{2}{|c|}{$\operatorname{MAL}\left\{\begin{array}{l}\text { Meaninglessness } \\
\text { Alienation }\end{array}\right.$} \\
\hline (Over) Nutrition & nd Identity \\
\hline (In) Activity & Occupation \\
\hline (Di) Stress & $\begin{array}{l}\text { Drugs, Alcohol and Smoking } \\
\text { Over-exposure }\end{array}$ \\
\hline Technology Induced Pathology & $\begin{array}{l}\text { Over-exposure } \\
\text { Under-exposure }\end{array}$ \\
\hline Inadequate Sleep & Relationships \\
\hline Environment & Social inequity \\
\hline
\end{tabular}

\section{Behind the Research

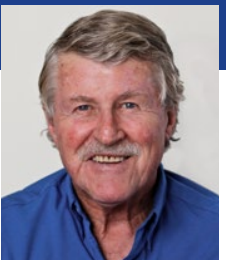 \\ Garry Egger

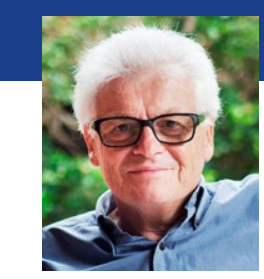 \\ Andrew Binns

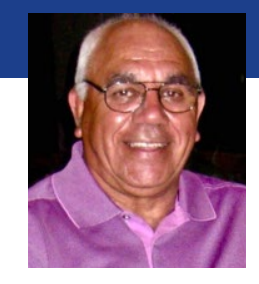 \\ Bob Morgan \\ E: eggergj@gmail.com T: 62408643914 W: www.lifestylemedicine.org.au}

\section{Research Objectives}

Understanding environmentally-related determinants of modern chronic diseases.

\section{Detail}

Address

Professor Garry Egger, 14 Arthur Street, Fairlight, NSW, Australia 2094

Bio

Garry Egger is a consultant in epidemiology. Andrew Binns is a GP in Lismore, Australia, John Stevens is a medical sociologist and consultant in Lifestyle Medicine, and Bob Morgan is a senor Aboriginal educator and consultant. All are members of the Australasian Society for Lifestyle Medicine (ASLM).

Collaborators

Australasian Society for Lifestyle Medicine.

Australasian Society of

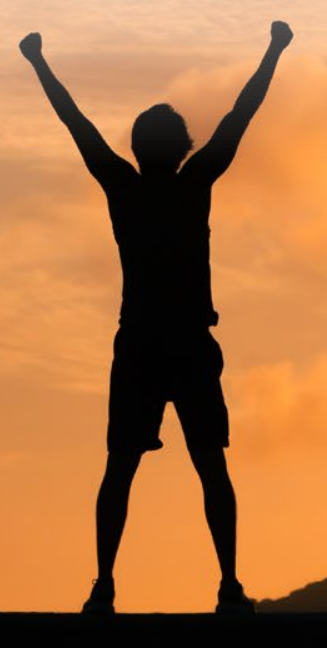

Lifestyle Medicine

\section{References}

Egger, G., Binns, A., \& Morgan, B. (2021). Adverse Childhood Clifostyle medicine do diog/10.1177/15598276211001292

Egger, G., Stevens, J., Binns, A., \& Morgan, B. (2019). Psychosocial Determinants of Chronic Disease: Implications for Lifestyle Medicine. American journal of lifestyle medicine, 13(6), 526-532.

doi.org/10.1177/1559827619845335

Egger G. (2016). Defining a Structure and Methodology for the Practice of Lifestyle Medicine. American journal of lifestyle medicine, 12(5), 396-403. doi.org/10.1177/1559827616669327

Egger, G., Colquhon, D. \& Dixon, J. (2013). 'Anthropogens' in Lfestyle Medicine. American journal of lifestyle medicine. doi.org/10.1177/1559827613512596

\section{Personal Response}

How can we take the next steps to understand more about the links between early life experiences and chronic ill health later in life?

II Typically, medical interventions for chronic disease (eg. factors related to the problem. Lifestyle Medicine expands this to include lifetime experiences and early psychosocial influences on later problems. ACES are now recognised this aetiolal dis in issues (eg. obesity) will be ineffectively managed. Experiences as "Upstream" Determinants of Lifestyle-Related 